Commentary

\title{
Neural correlates of temporality?
}

\section{Michał Klincewicz}

Philosophy and Cognitive Science, Graduate Center, City University of New York, 365 5th Avenue, New York, NY 10016, United States

\section{Introduction}

Dan Lloyd's “Neural Correlates of Temporality: Default Mode Variability and Temporal Awareness" has two goals. The main goal is to lay out the evidence for the neural correlate of temporality, which is Lloyd's term for the conscious experience of the passing of time. The secondary goal is to add "to an emerging science of consciousness an account of the thickness and effervescence of the extended Now" (Lloyd, this issue). ${ }^{1}$

The account of temporality Lloyd favors is Edmund Husserl's theory as it is developed in Phenomenology of Inner Time Consciousness. According to Husserl, temporality is a structural feature of all conscious experiences. On Husserl's view, a feature of conscious experience is structural when it is essential for the occurrence of any conscious experience at all. So temporality is not merely a part of the content of a conscious experience-it makes the presentations of conscious contents possible.

On Husserl's later view, temporality consists of three parts: retention, protention, and primal impression. Retention is a sort of short-term memory buffer that stores just-had conscious experiences. And protention is a buffer that stores conscious experiences one is about to have. Finally, primal impression is the cusp between retention and protention. Together these three components constitute a horizon on which conscious experiences appear to pass over time. And, according to Husserl, it is this retention-primal impression-protention structure that is essential for one to have any conscious experience at all. ${ }^{2}$

On this view, all conscious experiences, even those in retention, have the same structure. So the contents of retention have a retention-primal impression-protention structure themselves. And, through recursion, each of those stored structures itself has a retention-primal impression-protention structure in its retention component; which will itself contain a retention-primal impression-protention structure in its retention component, and so on.

On Husserl's view, any conscious experience contains in it a temporally ordered series of contents that were conscious before it. This implies that any conscious experience contains information about its temporal relation to conscious experiences that came before it.

\section{Lloyd's argument}

Whatever we make of Husserl's view, it sets the agenda for Lloyd's paper, which is to identify the neural correlate of temporality. Lloyd's strategy is to find brain activity that contains information about its temporal relation to preceding brain activity. Finding it, on Lloyd's view, would amount to finding the recursive structure of temporality posited by Husserl.

To find out whether any brain activity encodes information about activity that came before it, Lloyd makes use of programmable pattern recognizers. Lloyd first trains these programs in the task of detecting the temporal position of a particular fMRI image relative to others from the same series. Once this training is done, Lloyd feeds the pattern recognizers unordered fMRI images of off-task activity from an unrelated study. The pattern recognizers turn out to be quite good at sorting the temporal order of the off-task images. From this fact Lloyd concludes "something in the distributed pattern of component activity encodes elapsed time" (Lloyd, this issue).

\footnotetext{
Commentary on Lloyd, D. (2011). Neural correlates of temporality: Default mode variability and temporal awareness. Consciousness and Cognition, xx, $\mathrm{xxx}-\mathrm{xxx}$.

E-mail address: Michal.Klincewicz@gmail.com

1 All references to Lloyd (2011) in this volume unless noted otherwise.

${ }^{2}$ This is not a totally uncontroversial interpretation of Husserl's view, but it's the one that Lloyd himself appears to rely on Zahavi (2007, p. 468).
} 
Lloyd then analyzes the images with the goal of isolating the brain areas that encode temporal information recovered by the pattern recognizers. This analysis implicates regions previously identified as the Default Mode Network, which includes the medial prefrontal cortex, lateral temporal cortex, posteromedial cortex, and the inferior parietal cortex, among others. The most prominent areas in Lloyd's analysis are the medial prefrontal cortex, lateral temporal cortex, and hippocampal regions. So Lloyd concludes that these areas are the Dynamic Temporality Network (DTM), that is, the neural correlate of temporality. This is the master argument for the main conclusion of the paper.

\section{Levels of explanation}

I am skeptical about the significance of the performance of pattern recognizers. The images these computer programs learn to sort represent blood oxygen level-dependent contrasts, not contents. So it is not obvious why Lloyd thinks the programs' ability to sort these images indicates the presence of a recursive structure of contents.

One might be inclined to respond to this skepticism by urging that Husserl's notion of time-consciousness does not involve contents at all. After all, temporality is supposed to be a structural feature of consciousness, not a type of content. But this reply-whatever its merit in representing Husserl's view-changes the topic of conversation. Temporality is a feature of conscious experience that has a distinct phenomenology. It allows us to experience the passing of time and temporally extended objects such as melodies. Surely, if temporality makes a difference between hearing a melody and a mere sequence of tones, it features as a part of the content of our experience.

It also does not help that it is the "repetition of patterns (or at least pattern similarity) at different time points that drives the analysis" (Lloyd, this issue) of the pattern recognizers. We have no reason to suppose that the information in those patterns reflects any aspect of conscious experience. In short, neural level explanations do not amount to psychological level explanations-at least not without argument.

In order to connect neural level explanations with psychological level explanations Lloyd needs more than fMRI recordings and patterns. In the case of conscious experiences, which are Lloyd's concern, he needs first-person data such as subjective reports of the people whose brains are being scanned. I hope to find out why Lloyd thinks that in the case of temporality, voxel patterns are enough to build such a connection.

\section{Criteria for consciousness}

My skepticism about pattern recognizers comes from a more general worry about the paper. I find no compelling reason to think that Lloyd's data and analysis have anything to do with conscious experience at all-never mind a particular aspect of it such as temporality. As Lloyd himself acknowledges, "the alternative interpretation is that the DTN does encode elapsed time, but that information is not conscious" (Lloyd, this issue).

Anticipating this objection Lloyd points out that individual parts of the DTN have been shown to be involved in a variety of conscious experiences. In support of this claim Lloyd cites several studies of autobiographical memory retrieval, meta-cognition, and theory of mind. Unfortunately, this does not help much with the objection.

First of all, this response is too general. The cited studies concern a wide variety of tasks, none of which are the exclusive domain of conscious experience. While some philosophers tend to connect meta-cognition with consciousness, this view is not universal, and it is not explicitly mentioned in Lloyd's response. ${ }^{3}$ Consequently, we are left to wonder what these studies tell us about the involvement of the DTN in conscious experience. Instead of answering the objection, Lloyd's pointing to these results seems only to push the problem one step back.

In order to answer the objection Lloyd needs to state criteria that distinguish conscious mental states from those that are not conscious. Here, there are many views to choose from and I've already pointed to some that might be amenable to Lloyd's proposal. But until Lloyd adopts one of these criteria or states one of his own the question of what the DTN has to do with conscious experience will be left unanswered.

\section{Husserlian temporality and Lloyd's argument}

The second problem with Lloyd's response is that it is too specific. On the Husserlian conception of temporality all conscious experiences have the retention-primal impression-protention structure. So, if Lloyd is right in identifying the DTN as its neural correlate, we should expect it to be active whenever one has a conscious experience. But this appears not to be so.

It has been observed that during the performance of a goal-oriented task "specific brain functions unique to the baseline state itself are being temporarily suspended" (Raichle et al., 2001, p. 681). So brain areas that comprise the DTN show lower or no activation, even though the participants are having conscious experiences. This strongly suggests that the DTN cannot be the neural correlate of a ubiquitous feature of consciousness, such as temporality.

\footnotetext{
${ }^{3}$ One view that might be particularly compatible with Lloyd's hypothesis is the dispositional higher-order thought theory developed by Carruthers (2000). On that view, conscious mental states are accompanied by a disposition to have a higher-order thought about them. And this disposition is connected to one's ability to engage in theory of mind reasoning, which is correlated with activity in the medial prefrontal cortex (Saxe, Moran, Scholz, \& Gabrieli, 2006).
} 
One might respond in defense of Lloyd's thesis that the shutdown of the DTN actually comports with the phenomenology of conscious experience. Time flies when we are particularly engaged in doing something and we sometimes even seem to lose the sense of time altogether. So we should therefore expect the neural correlate of temporality to display diminished or no activation during the performance of goal-oriented tasks-especially if we are particularly engaged in those tasks.

But the metaphors are misleading. Being particularly engaged in a task does not involve diminished conscious experience of the passing of time or an absence of conscious experience altogether. The more accurate phenomenological description is that time appears to pass faster. Just as when time drags on-as is presumably the case when people are stuck inside of an fMRI machine for too long-conscious experience misrepresents the rate of the flow of time.

Finally, there is some indirect evidence that the DTN cannot be the neural correlate of a ubiquitous aspect of conscious experience such as temporality. The areas composing the Default Mode Network show marginal functional integration in children under 9 years old (Fair et al., 2008). But young children have conscious experiences of the passing of time, even if it might appear to them to be passing by less quickly than it does when they are adults (Crawley \& Pring, 2000).

The impact of the relative lack of functional integration of the Default Mode Network does not manifest itself in the child's conscious experience of the passing of time, but their performance on a variety of higher cognitive tasks such as theory of mind, reference to self, and autobiographical memory retrieval. This shows that the part of the Default Mode Network Lloyd identifies as the DTN drives performance, but does not play an equally important role in a ubiquitous feature of conscious experience such as temporality.

\section{Conclusion}

All of this makes it difficult to accept Lloyd's master argument. We must conclude that it falls short of reaching the main goal of the paper. But that does not mean that the secondary goal has to be abandoned as well. If we accept the results from the performance of pattern recognizers, Lloyd gives new evidence about "which brain regions might be particularly involved in encoding temporal information" (Lloyd, this issue).

\section{Acknowledgment}

Thanks to Valtteri Arstila for helpful conversations about some of the ideas presented here.

\section{References}

Carruthers, P. (2000). Phenomenal consciousness: A naturalistic theory. Cambridge University Press.

Crawley, S. E., \& Pring, Linda (2000). When did Mrs. Thatcher resign? The effects of ageing on the dating of public events. Memory, 8(2), 111-121.

Fair, D. A., Cohen, A. L., Dosenbach, N. U. F., Church, J. A., Miezin, F. M., Barch, D. M., et al (2008). The maturing architecture of the brain's default network. Proceedings of the National Academy of Sciences, 105(10), 4028-4032.

Lloyd, D. (2011). Neural correlates of temporality: Default mode variability and temporal awareness. Consciousness and Cognition. doi:10.1016/ j.concog.2011.02.016.

Raichle, M. E., MacLeod, A. M., Snyder, A. Z., Powers, W. J., Gusnard, D. A., \& Shulman, G. L. (2001). A default mode of brain function. Proceedings of the National Academy of Sciences of the United States of America, 98(2), 676-682.

Saxe, R., Moran, J. M., Scholz, J., \& Gabrieli, J. (2006). Overlapping and non-overlapping brain regions for theory of mind and self reflection in individual subjects. Social Cognitive and Affective Neuroscience, 1(3), 229-234.

Zahavi, D. (2007). Perception of duration presupposes duration of perception-or does it? Husserl and Dainton on time. International Journal of Philosophical Studies, 15(3), 453-471. 